\title{
Reduced Klotho expression contributes to poor survival rates in human patients with ovarian cancer, and overexpression of Klotho inhibits the progression of ovarian cancer partly via the inhibition of systemic inflammation in nude mice
}

\author{
YOULIANG YAN ${ }^{1}$, YIFENG WANG $^{2}$, YI XIONG $^{1},{\text { XIUFENG } \text { LIN }^{1}, \text { PING ZHOU }^{1} \text { and ZHIYING CHEN }}^{1}$ \\ ${ }^{1}$ Department of Obstetrics and Gynecology, Boai Hospital of Zhongshan of Southern Medical University, Guangzhou, \\ Guangdong 528403; ${ }^{2}$ Department of Obstetrics and Gynecology, Zhujiang Hospital of Southern Medical University, \\ Guangzhou, Guangdong 510282, P.R. China
}

Received July 29, 2015; Accepted July 25, 2016

DOI: $10.3892 / \mathrm{mmr} .2017 .6172$

\begin{abstract}
Klotho is a recently discovered anti-aging gene, which has been reported as a tumor suppressor in numerous human malignancies; however, the role of Klotho in human ovarian cancer remains to be elucidated. The aim of the present study was to detect the expression of Klotho and evaluate its association with the progression of human ovarian cancer. A clinical follow-up study of 120 patients with ovarian cancer and 78 normal controls was conducted. The expression levels of Klotho were determined by western blotting and immunohistochemistry. The results demonstrated that high Klotho expression levels were detected in all normal controls, whereas the positive rate of Klotho was $61.6 \%$ in the ovarian cancer group, which was significantly decreased compared with in the control group $(\mathrm{P}<0.01)$. Furthermore, reduced Klotho expression was significantly correlated with decreased survival rates in patients with ovarian cancer $(\mathrm{P}=0.025)$. Subsequently, Klotho levels were detected in seven human ovarian cancer cell lines by western blotting. The results demonstrated that the highest levels of Klotho were detected in $\mathrm{CaOV} 3$ cells, medium levels of Klotho were detected in CaOV4 and SKOV-3 cells, and almost no Klotho was detected in the other four cell lines: OVCA 432, OVCAR-5, OVCAR-8 and A2780 cells. The association between Klotho levels and cell proliferation was determined by MTT assay, and the results indicated that higher levels of Klotho inhibited the proliferation of A2780 and OVCAR-5 cells, whereas reduced Klotho expression promoted cell growth of $\mathrm{CaOV} 3$ and SKOV-3 cells. In addition,
\end{abstract}

Correspondence to: Mr. Yifeng Wang, Department of Obstetrics and Gynecology, Zhujiang Hospital of Southern Medical University, 253 Industrial Avenue, Haizhuqu, Guangzhou, Guangdong 510282, P.R. China

E-mail:wangayifeng@hotmail.com

Key words: Klotho, ovarian cancer, interleukin-6, interleukin-1 $\beta$ the plasma levels of inflammatory cytokines in tumor-bearing mice and normal control mice were detected by enzyme-linked immunosorbent assay, and plasma interleukin (IL)-6 and IL-1 $\beta$ levels were elevated in all tumor-bearing mice. Notably, the mRNA expression levels of IL-6 were significantly higher in the liver, ovaries and kidneys of $\mathrm{Klotho}^{-/}$mice compared with in wild type mice $(\mathrm{P}<0.01)$, thus indicating that aberrant Klotho expression may contribute to systemic inflammation in $\mathrm{Klotho}^{--}$mice. Finally, the in vivo antitumor role of aberrant Klotho expression was determined in a nude mice model. A2780 cells were transfected with pCMV6-Klotho and the stably transfected cells were screened; the mice were injected with the stably transfected cells. The results indicated that tumor volume and tumor weight were significantly decreased in the pCMV6-Klotho group compared with in the pCMV6 vector group $(\mathrm{P}<0.01)$. These findings suggest that overexpression of Klotho may suppress tumor growth in animal models. In conclusion, Klotho was demonstrated to act as a potent tumor suppressor in human ovarian cancer cells. Reduced Klotho expression was detected in the specimens of patients with ovarian cancer, and overexpression of Klotho significantly inhibited cell proliferation of human ovarian cancer cells. Therefore, Klotho may be considered a useful key target for the molecular therapy of human ovarian cancer.

\section{Introduction}

Ovarian cancer is a common malignancy of the female reproductive organs $(1,2)$. The incidence of ovarian cancer ranks third, after cervical cancer and uterine body cancer; however, the mortality rate is the highest compared with all other types of gynecological tumor, and it is therefore a serious threat to survival (3-5). In the United States, it was estimated that 22,240 cases of ovarian cancer were newly diagnosed, and an estimated 14,030 cases of mortality were associated with ovarian cancer in 2013 (6,7). The major underlying cause for the high mortality rate is that $\sim 75 \%$ of women had metastases throughout the peritoneal cavity and were diagnosed with ovarian cancer at an advanced stage. Although combined 
chemotherapy may improve the overall survival rate in patients with advanced stage ovarian cancer, novel therapeutic paradigms are required. Furthermore, the underlying molecular mechanism remains to be clearly defined, since the pathology differs in various types of ovarian cancer.

Klotho is a recently discovered anti-aging protein, and Klotho-deficient mice exhibit a premature aging-like syndrome (8). Klotho is predominantly distributed in the kidneys and brain, and has an essential role in protecting against dysfunction of the kidney-brain axis during the aging process (9). There are two types of Klotho protein: Transmembrane and secreted forms of Klotho (10,11). Both of these proteins exert distinct functions, which may collectively affect aging processes in mammals. The single-pass transmembrane protein forms a complex with numerous fibroblast growth factor receptors, whereas secreted Klotho protein regulates the activity of several ion channels and growth factors, including insulin, insulin-like growth factor 1 (IGF-1) and Wnt (12). Klotho has been studied and reported to act as a tumor suppressor in various human malignancies (13-15). Previous studies have focused on the role of Klotho in tumorigenesis, cancer progression and prognosis. Klotho has been reported to exert antitumor effects by inhibiting insulin/IGF1, p53/p21 and Wnt signaling, and silencing Klotho expression was mediated by promoter hypermethylation and histone deacetylation in the progression of tumors $(13,16)$. In addition, Lee et al examined epigenetic silencing of Klotho in human cervical carcinoma, and the functional loss of Klotho as a secreted Wnt antagonist contributed to aberrant activation of the canonical Wnt pathway in cervical carcinoma (17).

To the best of our knowledge, there are currently two main research groups that have reported on the role of Klotho in human ovarian cancer. Lu et al demonstrated that Klotho expression was associated with epithelial ovarian cancer progression and the protein could serve as an independent marker for the prognosis of ovarian cancer (18). Lojkin et al reported that Klotho acted as an inhibitor of the IGF-1 pathway in cancer cells; and restoring its expression slowed the proliferation of epithelial ovarian cancer cells and inhibited transcriptional activity of the estrogen receptor (13). The present study aimed to clarify the association between the expression levels of Klotho in human ovarian cancer tissues and the progression of ovarian cancer. Furthermore, the molecular mechanism underlying the effects of Klotho on ovarian cancer cell lines was explored. The present study provided novel evidence regarding the molecular mechanism underlying human ovarian cancer.

\section{Materials and methods}

Patients. Patients were recruited from the Zhujiang Hospital of Southern Medical University (Guangzhou, China). A total of 120 patients with ovarian cancer and 78 normal controls were recruited to the present study. The current study was conducted over a period of 36 months, between March 2012 and March 2015. All patients were diagnosed with ovarian cancer and underwent surgery following their diagnosis. The median age of the patients was 56.8 years (range, 26-82 years). The paired paracancerous tissues were collected as normal controls. The patient was diagnosed with ovarian cancer using a pathological diagnosis. They have not received chemotherapy or irradiation prior to tissue collection. Fresh tumor tissues were collected from each patient during surgery. One fresh tissue sample from each patient was frozen at $-80^{\circ} \mathrm{C}$ for western blotting. Another tissue sample from each patient was fixed in $10 \%$ formalin, and paraffin-embedded sections were prepared and cut into $4 \mu \mathrm{m}$ sections. The research carried out on humans was in compliance with the Helsinki Declaration, and the present study was approved by the Ethics Committee of Zhujiang Hospital of Southern Medical University. The subjects were well informed of the details and signed relevant contracts prior to the study.

Cell lines and reagents. The A2780, SKOV-3, OVCA 432, OVCAR-5 OVCAR-8, CaOV4 and CaOV3 human ovarian cancer cell lines were obtained from the American Type Culture Collection (Manassas, VA, USA). Dulbecco's modified Eagle's medium (DMEM) and fetal bovine serum (FBS) were purchased from Hyclone (GE Healthcare Life Sciences, Logan, UT, USA). Total RNA extraction kit (cat. no. MK700) and cDNA reverse transcription kit (cat. no. 6110) were obtained from Takara Bio, Inc. (Kusatsu, Japan). Lipofectamine 2000 (cat. no. 11668-019) was obtained from Invitrogen (Thermo Fisher Scientific, Inc., Waltham, MA, USA). The stock concentration of short hairpin (sh)RNA was $20 \mu \mathrm{M}$ and the working concentration was $50 \mathrm{nM}$. Klotho shRNA Plasmid and control shRNA Plasmid-A were obtained from Santa Cruz Biotechnology Inc. (Dallas, TX, USA). A2780 cells were plated into a 24 -well plate at a density of $2 \times 10^{4}$ cells/well. The cells were grown to $70-80 \%$ confluence. Klotho shRNA plasmid $(2 \mu \mathrm{l})$ or control shRNA plasmid $(2 \mu \mathrm{l})$ and $5 \mu \mathrm{l}$ Lipofectamine 2000 were diluted into $100 \mu \mathrm{l}$ Opti-MEM, respectively. The shRNA were gently mixed and maintained at room temperature for $5 \mathrm{~min}$. Next, the $100 \mu \mathrm{l}$ of Opti-MEM containing Klotho shRNA and $100 \mu \mathrm{l}$ Opti-MEM containing lipofectamin 2000 were gently mixed together and kept for $15 \mathrm{~min}$ at room temperature. Finally, the mixture was added into the medium of the human ovarian cancer cells to knockdown the Klotho expression levels for indicated time.

Antibodies. Rabbit polyclonal anti-Klotho antibody (cat. no. ab18131) was purchased from Abcam (Cambridge, MA, USA) for use in western blotting. Anti-Klotho antibody (E-21; cat. no. sc-22220) was obtained from Santa Cruz Biotechnology, Inc. for use in immunohistochemistry. Mouse monoclonal anti- $\beta$-actin antibody (cat. no. TA310155) was obtained from OriGene Technologies, Inc.(Beijing, China). The secondary antibodies, including goat anti-rabbit immunoglobulin (Ig) G-horseradish peroxidase (HRP) (cat. no. sc-2004) and goat anti-mouse IgG-HRP (cat. no. sc-2005) were obtained from Santa Cruz Biotechnology, Inc.

Immunohistochemical analysis. Immunohistochemical analysis was performed as described previously (19,20). Briefly, paraffin-embedded sections were dewaxed, rehydrated, blocked and incubated overnight at $4^{\circ} \mathrm{C}$ with the primary antibody specific to Klotho. After three washes with phosphate-buffered saline (PBS), the sections were incubated with secondary antibody for $1 \mathrm{~h}$ at room temperature. Subsequently, the slides were dehydrated, mounted in Permount and visualized under 
Table I. Klotho expression in human ovarian cancer tissues and normal ovarian tissues.

\begin{tabular}{lrrrr}
\hline & & \multicolumn{2}{c}{ Klotho } & \\
\cline { 3 - 3 } Tissue type & \multicolumn{1}{c}{$\mathrm{n}$} & + & - & $\begin{array}{l}\text { Positive } \\
\text { rate (\%) }\end{array}$ \\
\hline Normal gastric tissues & 78 & 78 & 0 & 100.0 \\
Gastric cancer tissues & 120 & 74 & 46 & $61.6^{\mathrm{a}}$ \\
\hline
\end{tabular}

${ }^{\text {a }}<0.01$, compared with the normal ovarian tissues.

a Nikon Eclipse Ti microscope (Nikon Corporation, Tokyo, Japan). Positive and negative images were captured using a camera attached to the microscope at 400x magnification.

Western blot analysis. Frozen cancerous and paracancerous tissues were homogenized using TRIzol reagent (Invitrogen; Thermo Fisher Scientific, Inc.) using the PowerGen 125 homogenizer (Thermo Fisher Scientific, Inc.). The samples were washed with PBS and lysed with radioimmunoprecipitation assay buffer [50 mmol/l Tris, $1 \% \mathrm{NP}-40,150 \mathrm{mmol} / \mathrm{l}$ $\mathrm{NaCl}, 1 \mathrm{mmol}$ EDTA, $0.1 \%$ sodium dodecyl sulfate (SDS), $0.25 \%$ SDC]. Whole protein concentrations were quantified using the Bradford assay. Proteins ( $20 \mu \mathrm{g}$ per lane) were then separated by $10 \%$ SDS-polyacrylamide gel electrophoresis and were transferred electrophoretically to a nitrocellulose membrane at $400 \mathrm{~mA}$ for $1 \mathrm{~h}$. The membrane was blocked with 5\% non-fat milk in Tris-buffered saline containing Tween (TBST; $50 \mathrm{mmol} / 1$ Tris- $\mathrm{HCl}, 150 \mathrm{mmol} / \mathrm{l} \mathrm{NaCl}$ and $0.1 \%$ Tween). Subsequently, the membranes were incubated with primary antibodies at $4^{\circ} \mathrm{C}$ overnight and secondary antibodies for $40 \mathrm{~min}$ at room temperature. Between incubations the membranes were washed with TBST for $5 \mathrm{~min}$. The bands were detected using an enhanced chemiluminescence western blotting detection system, according to the manufacturer's protocol. $\beta$-actin was used as an internal reference. The bands were developed by ECL chemiluminescence kit and visualized by gel imaging system (Bio-Rad Laboratories, Inc., Hercules, CA, USA). Image J version 1.49 (National Institutes of HealthBethesda, MD, USA) was used to analyze the results form western blotting.

3-(4,5-dimethylthiazol-2-yl)-2,5-diphenyltetrazolium bromide (MTT) assay. The cells were cultured in DMEM, supplemented with $10 \% \mathrm{FBS}, 37^{\circ} \mathrm{C}$ in $5 \% \mathrm{CO}_{2}$ atmosphere. An MTT assay was performed as described previously $(21,22)$. Briefly, the human ovarian cancer cell lines were transfected with pCMV6-Klotho or pCMV6 vector (both obtained from OriGene Technologies, Inc.) for 48, 72 and $96 \mathrm{~h}$. The cancer cells $\left(1 \times 10^{5}\right.$ cells/well) were seeded into a 48 -well plate. The cells were grown to $70-80 \%$ confluence. The vector (100 ng) and $5 \mu \mathrm{l}$ Lipofectamine 2000 were diluted into $100 \mu \mathrm{l}$ of Opti-MEM which were maintained for $5 \mathrm{~min}$ at room temperature. They were mixed gently for $15 \mathrm{~min}$. The mixture was added into the medium of human ovarian cancer cells and cultured for the aforementioned times. Cell proliferation in each group was detected by MTT assay.

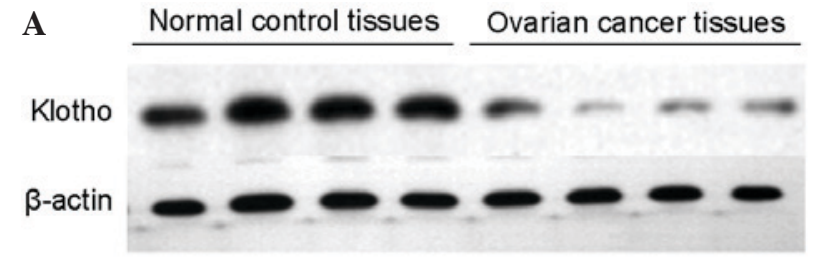

B

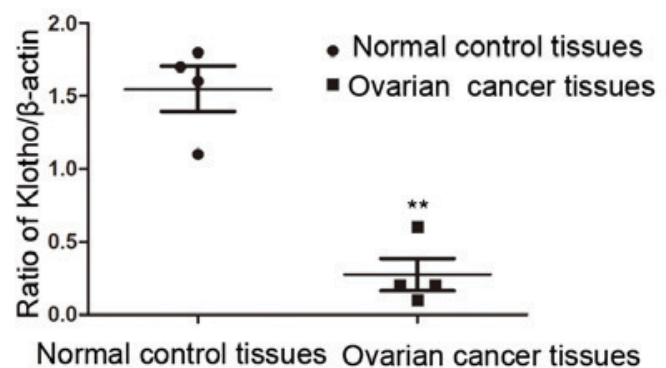

Figure 1. Klotho is significantly decreased in human ovarian cancer tissues compared with in normal control tissues. (A) Expression levels of Klotho were detected by western blotting. Data are representative of four independent specimens. The housekeeping gene $\beta$-actin was used as an internal reference in the experiment. (B) Ratios of Klotho/ $\beta$-actin are presented as a grouped column scatter graph. The bands of expressed Klotho were captured and analyzed by ImageJ software. The expression levels of Klotho were normalized to $\beta$-actin. Data are presented as the mean \pm standard deviation. ${ }^{* * *} \mathrm{P}<0.01$ compared with the normal control tissues.

Enzyme-linked immunosorbent assay (ELISA). Whole blood samples $(0.2 \mathrm{ml})$ were obtained from the tail veins of the mice and were maintained at room temperature for $2 \mathrm{~h}$, and the supernatant was obtained following centrifugation at $1,000 \mathrm{x} \mathrm{g}$ for $20 \mathrm{~min}$ at room temperature. The concentration of inflammatory cytokines was determined using human IL-6 and IL-1 $\beta$ ELISA kits (NeoBioscience, Beijing, China) according to the manufacturer's protocols. Absorbance was measured at $450 \mathrm{~nm}$ using a Benchmark Microplate Reader (Bio-Rad Laboratories, Inc., Hercules, CA). All of the samples were analyzed in duplicate for cytokine levels. The concentrations of interleukin (IL)- 6 and IL- $1 \beta$ in the samples were determined from standard curves.

Reverse transcription-polymerase chain reaction. Total RNA was isolated from the tissues or cultured cells using TRIzol RNA regent (Tiangen Biotech Co., Ltd., Beijing, China). RNA $(1 \mu \mathrm{g})$ was reverse transcribed by reverse transcriptase SuperScript III (Invitrogen; Thermo Fisher Scientific, Inc.). The primer sequence of Klotho was sense 5'-ACCTGGTGG CGCACAACC-3' and antisense 5'-TTGGCAAACCAACCT AGTACA-3'. The PCR reaction conditions were as follows: $94^{\circ} \mathrm{C}$ for $5 \mathrm{~min}$, followed by 30 cycles of $94^{\circ} \mathrm{C}$ for $30 \mathrm{sec}, 55^{\circ} \mathrm{C}$ for $30 \mathrm{sec}$ and $72^{\circ} \mathrm{C}$ for $30 \mathrm{sec}$ and finally, elongation at $72^{\circ} \mathrm{C}$ for $10 \mathrm{~min}$.

Animals. Male C57BL/6 mice ( $\mathrm{n}=18$; weight, $18-20 \mathrm{~g}$; age, 6-8 weeks) were obtained from the Laboratory Animal Center of Peking University Health Science Center (Beijing, China) and were maintained in the specific pathogen-free conditions. The temperature was controlled at $18-22^{\circ} \mathrm{C}$, humidity of 50-60\%. Food and water was provided ad libitum. The light:dark cycle was 12:12 h. The mice were challenged 
A

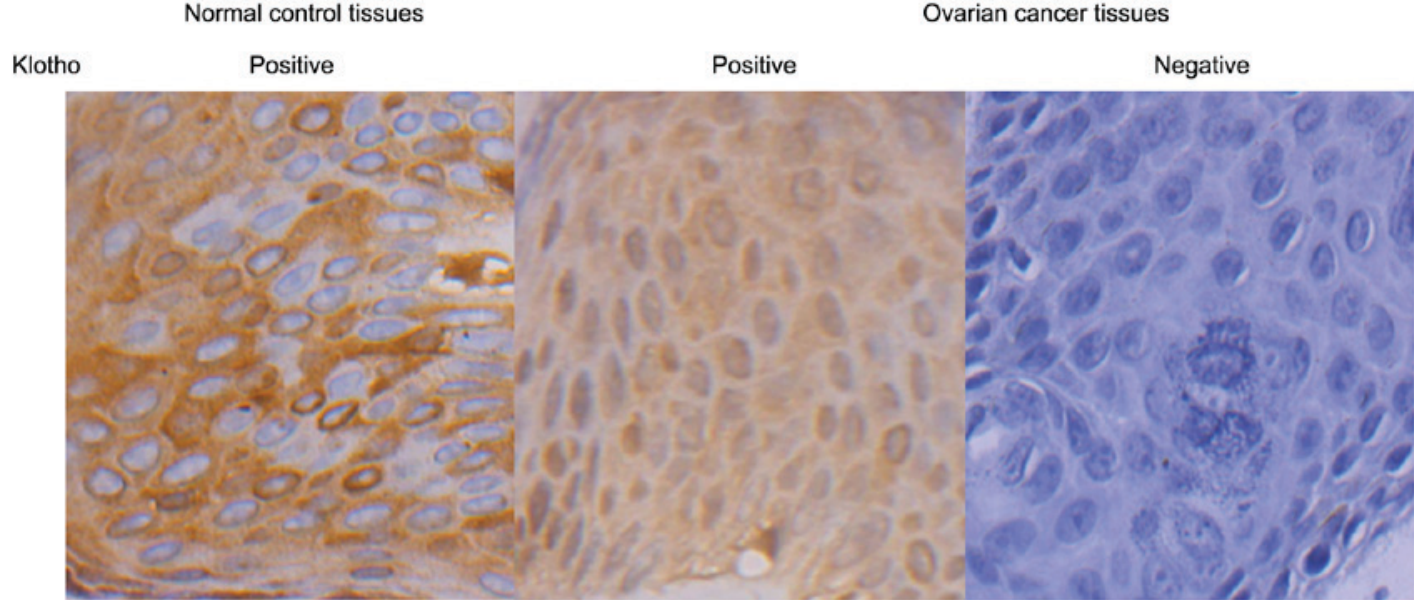

B

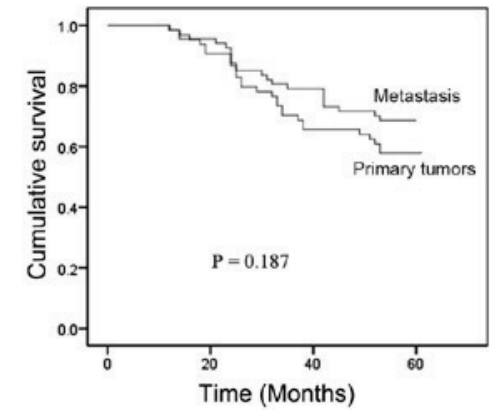

C

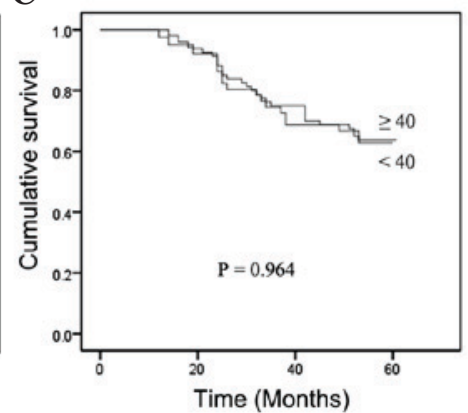

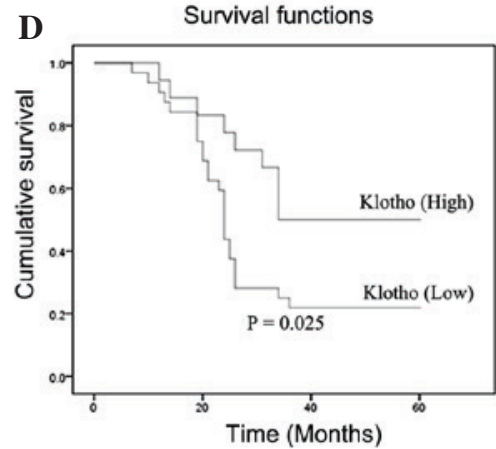

Figure 2. Survival rate is positively correlated with Klotho expression in patients with ovarian cancer. (A) Immunohistochemical analysis of Klotho. Specimens from 120 patients with ovarian cancer and 78 normal controls were collected. Positive Klotho staining was detected in normal control tissues. In patients with ovarian cancer, positive and negative staining of Klotho was detected in the collected specimens. Images of Klotho staining analysis were captured at 400x original magnification. (B) Survival analysis between patients with primary and metastatic tumors. Kaplan-Meier curves revealed there was no statistically significant difference in survival rate between patients with primary tumors and metastasis $(\mathrm{P}=0.187)$. (C) Survival analysis between patients that were $<40$ years old and those that were $\geq 40$ years old. Kaplan-Meier curves revealed there was no statistically significant difference in survival rate between patients $<40$ years old and patients $\geq 40$ years old ( $\mathrm{P}=0.964)$. (D) Patients with ovarian cancer were separated into those with high levels of Klotho [Klotho (high)] and those with low levels of Klotho [Klotho (low)]. Kaplan-Meier analysis detected a significantly decreased survival rate in patients with lower Klotho expression ( $\mathrm{P}=0.025)$.

subcutaneously with $4 \times 10^{4}$ A2 2780 cells in the flank area; each group contained six mice. For the in vivo antitumor experiment, the mice were randomly divided into three groups: i) Klotho group; ii) control plamid group and iii) negative control group. $\mathrm{Klotho}^{-/-}$mice ( $\mathrm{n}=18$; weight, 18-20 g; age, 8 weeks) were obtained from CasGene Biotech Co., Ltd. (Beijing, China) and housed in specific pathogen-free conditions. The temperature was controlled at $18-22^{\circ} \mathrm{C}$, humidity of $50-60 \%$. Food and water was provided ad libitum. The light:dark cycle was 12:12 h. Wild type mice with the same genetic background were used as a negative control. After 2 weeks, cytokine levels in the serum were detected by ELISA. The animals used in the present study received humane care in compliance with the Guide to the Care and Use of Experimental Animals formulated by the Medical Ethical Committee on animal experiments of Zhujiang Hospital of Southern Medical University. The mice were sacrificed by cervical after 1 month of tumor injections. The mice were disinfected with $75 \%$ alcohol. The tumors in nude mice were weighed and compared between the different groups.

Statistical analysis. Statistical analysis was performed using SPSS 20.0 software (IBM SPSS, Armonk, NY, USA). data are presented as the mean \pm standard deviation. Comparisons
A

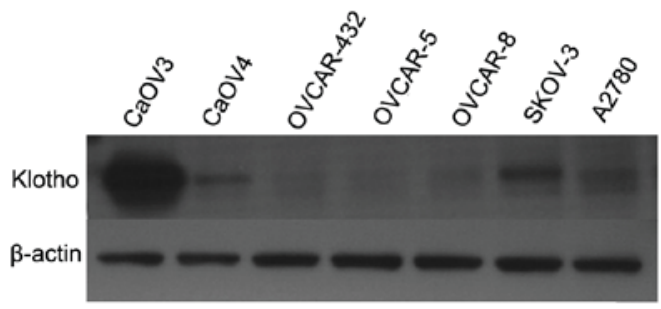

B

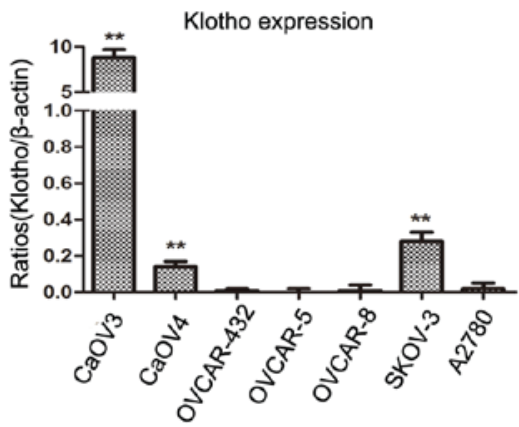

Figure 3. Expression levels of Klotho were detected in ovarian cancer cell lines. (A) Klotho protein levels were determined by western blotting in human ovarian cancer cell lines. $\beta$-actin was used as the internal reference in $\mathrm{CaOV} 3, \mathrm{CaOV} 4$, OVCA 432, OVCAR-5, OVCAR-8, SKOV-3 and A2780 cells. (B) Histogram of relative expression levels in human ovarian cancer cell lines. ${ }^{* *} \mathrm{P}<0.01$, compared with Klotho expression in A2780 cells. 


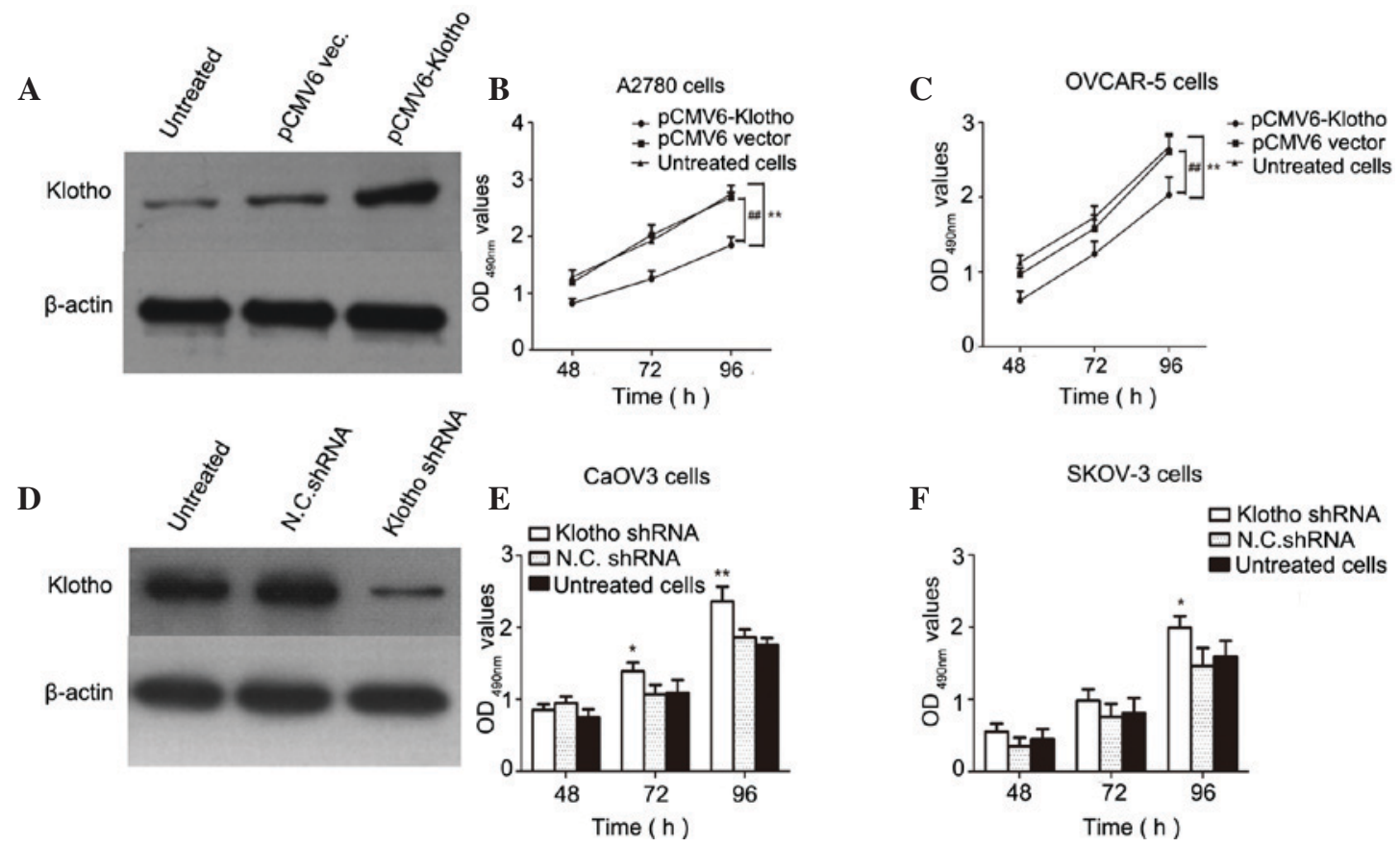

Figure 4. High levels of Klotho inhibit the proliferation of human ovarian cancer cells. (A) Human ovarian cancer cells were transfected with a pCMV6-Klotho expression vector or a control pCMV6 vector (vec.). Expression levels were determined by western blotting. (B) A2780 and (C) OVCAR-5 cells with low Klotho expression were transfected with pCMV6-Klotho. The cells were plated into 96-well plates and an MTT assay was used to determine the proliferation of pCMV6-Klotho and pCMV6 vector-transfected cells. ${ }^{* *} \mathrm{P}<0.01$ vs. untreated cells; ${ }^{\# \#} \mathrm{P}<0.01$ vs. pCMV6 vector-transfected cells. (D) Ovarian cancer cells were transfected with Klotho short hairpin (sh)RNA or negative control (N.C.) shRNA for 48 h. Western blotting was used to determine Klotho expression. (E) CaOV3 and (F) SKOV-3 human ovarian cancer cell lines with high levels of Klotho were transfected with Klotho-specific shRNA for 48, 72 and 96 h. Cell proliferation was determined by MTT assay. ${ }^{*} \mathrm{P}<0.05,{ }^{* *} \mathrm{P}<0.01$, compared with N.C. shRNA-transfected cells. OD, optical density.

A

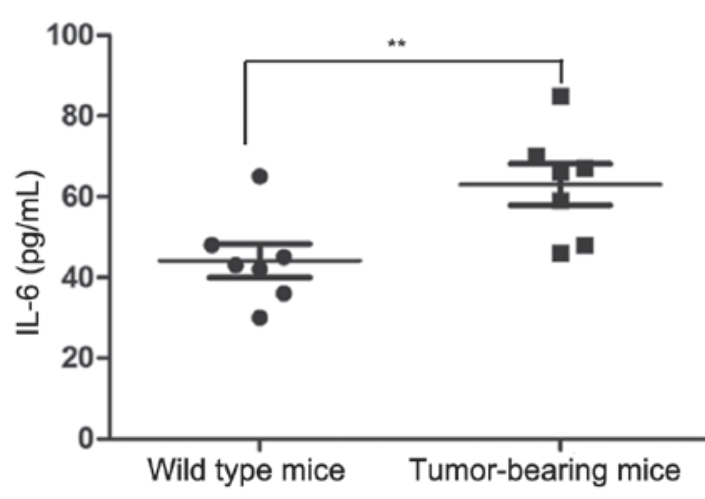

B

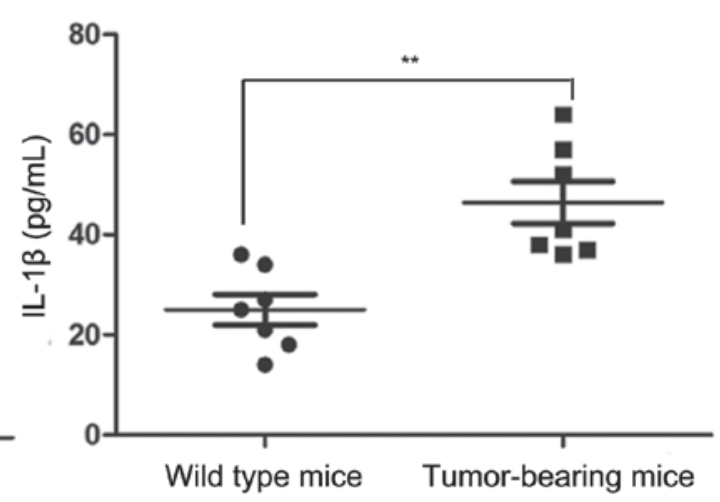

Figure 5. Plasma interleukin (IL)-6 and IL-1 $\beta$ levels are elevated in all tumor-bearing mice. Male C57BL/6 mice were randomly divided into two groups ( $\mathrm{n}=6$ /group). The mice were challenged intraperitoneally with $4 \times 10^{4} \mathrm{~A} 2780$ cells in the flank area. After 2 weeks, serum samples from each mouse were prepared and the plasma concentrations of (A) IL-6 and (B) IL-1 $\beta$ were detected by enzyme-linked immunosorbent assay. " P<0.01.

between Klotho expression levels and clinical indicators were performed by $\chi^{2}$ test. Analysis of variance (ANOVA), followed by a post-hoc test was used for the comparison of multiple groups. A repeated measures ANOVA was used to compare tumor size in mice over time. Kaplan-Meier survival estimates were used to evaluate survival rate. $\mathrm{P}<0.05$ was considered to indicate a statistically significant difference.

\section{Results}

Expression levels of Klotho in human ovarian cancer tissues and normal ovarian tissues. In order to identify the role of
Klotho in the progression of human ovarian cancer, the expression levels of Klotho were detected by western blotting in patients with human ovarian cancer and normal controls. A total of 120 ovarian cancer specimens and 78 normal ovarian specimens were collected from the hospital; the median age of the patients was 56.8 years (range, 26-86 years). It was essential to use an appropriate internal reference in the experiment; therefore, $\beta$-actin was used as the internal reference, since this housekeeping gene exhibits stable expression in various types of cells and tissues. The data were normalized to $\beta$-actin and are presented as the mean \pm standard deviation. As presented in Fig. 1, the expression levels of Klotho were significantly 
A

Serum

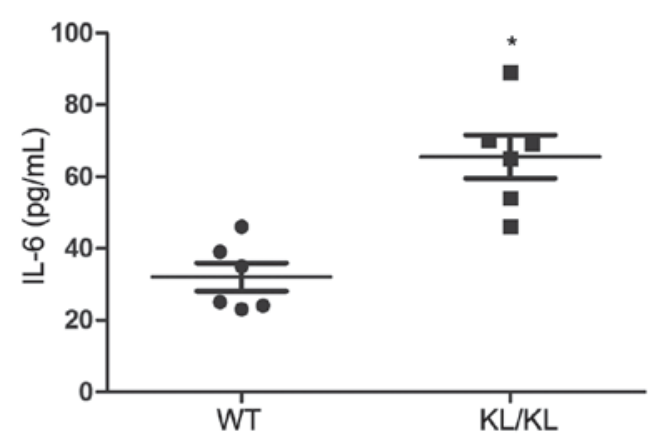

C

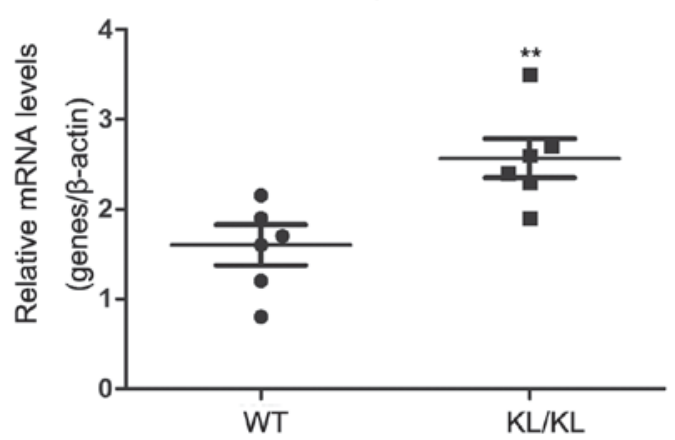

B

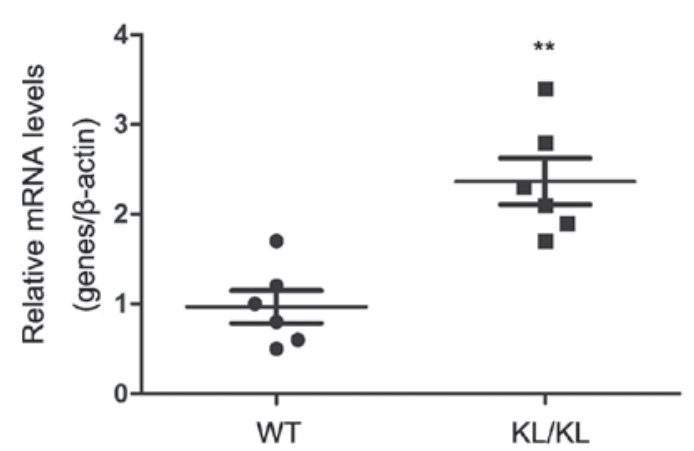

D

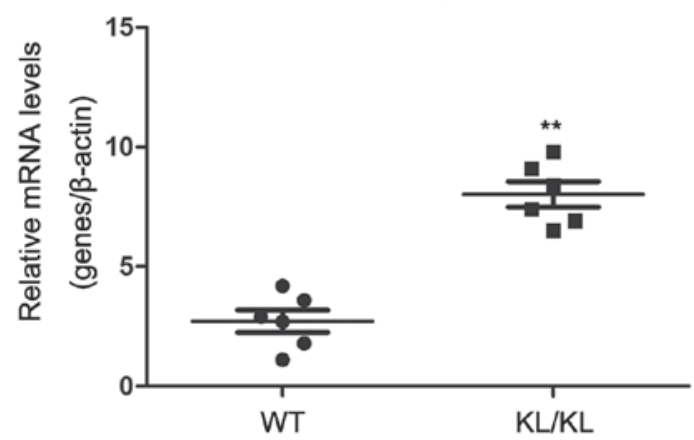

Figure 6. Aberrant Klotho expression contributes to systemic chronic inflammation in Klotho ${ }^{-/}(\mathrm{KL} / \mathrm{KL})$ mice. KL/KL mice (age, 8 weeks) and wild type (WT) mice with the same genetic background were investigated. (A) Concentration of interleukin (IL)- 6 in the serum was determined by enzyme-linked immunosorbent assay. (B-D) Expression was quantified by reverse transcription-quantitative polymerase chain reaction (RT-qPCR). Total RNA was extracted from the tissues and the mRNA levels of IL-6 in the (B) liver, (C) ovaries and (D) kidneys were detected by RT-qPCR. Each group randomly contained six mice. ${ }^{*} \mathrm{P}<0.05,{ }^{* *} \mathrm{P}<0.01$.

decreased in human patients with ovarian cancer compared with in the normal control group $(\mathrm{P}<0.01)$.

Survival rate is positively correlated with Klotho levels in patients with ovarian cancer. As shown in Fig. 2A, formalin-fixed, paraffin-embedded tissues from 120 patients with ovarian cancer and 78 normal controls were analyzed by immunohistochemistry to detect the protein expression of Klotho. The results indicated that positive Klotho expression was detected in all normal ovarian specimens; however, in human ovarian cancer patients, the positive rate of Klotho was $61.6 \%$, which was significantly decreased compared with in the normal control group (Table I).

In order to clarify the relevance of clinical parameters in the prognosis of human ovarian cancer, the relationship between clinical indicators, including age, metastasis and Klotho expression, and the 5-year survival rates of patients with ovarian cancer was determined. As shown in Fig. 2B, Kaplan-Meier curves demonstrated the survival rate was higher in patients with primary tumors compared with those with metastasis; however, there was no statistical difference $(\mathrm{P}=0.187)$. Kaplan-Meier curves were also generated to compare the survival rates of patients that were $<40$ years old with those that were $\geq 40$ years old; the results demonstrated that there was no statistically significant difference between the two groups ( $\mathrm{P}=0.964 ;$ Fig. $2 \mathrm{C}$ ). In addition, reduced Klotho expression was significantly correlated with decreased survival rates in patients with ovarian cancer $(\mathrm{P}=0.025$; Fig. $2 \mathrm{D})$. These results indicated that Klotho expression was associated with a higher survival probability in patients with human ovarian cancer.

Klotho expression levels in ovarian cancer cell lines. The expression levels of Klotho were detected in several human ovarian cancer cell lines by western blotting. As shown in Fig. 3, Klotho protein was not expressed or was expressed at low levels in four cell lines: OVCA 432, OVCAR-5, OVCAR-8 and A2780. Low expression levels were observed in two cell lines: CaOV4 and SKOV-3. Notably, the CaOV3 human ovarian cancer cell line expressed high levels of Klotho compared with in the ovarian cancer cell lines where no Klotho was detected $(\mathrm{P}<0.01)$.

High levels of Klotho inhibit the proliferation of human ovarian cancer cells. In order to clarify the relationship between Klotho expression and the proliferation of ovarian cancer cells, two human ovarian cancer cell lines were selected as a cell model. A2780 cells exhibited hardly any detectable Klotho expression, and $\mathrm{CaOV} 3$ cells exhibited high Klotho levels. In Fig. 4A, Klotho was overexpressed in A2780 cells and the results demonstrated that Klotho levels were markedly increased compared with in the negative control cells. Subsequently, proliferative activity was determined by MTzT assay in pCMV6-Klotho and pCMV6 vector-transfected ovarian cancer cells. The data revealed that overexpression of Klotho in A2780 and OVCAR-5 cells contributed to the inhibition of human ovarian cancer cell proliferation compared with in untreated and pCMV6 
A

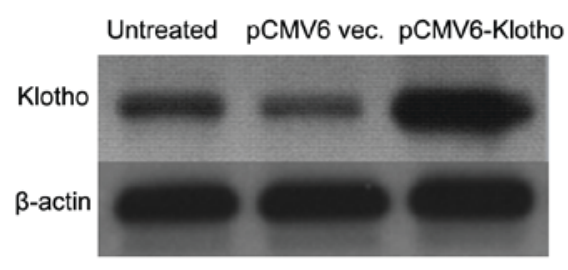

$\mathbf{C}$

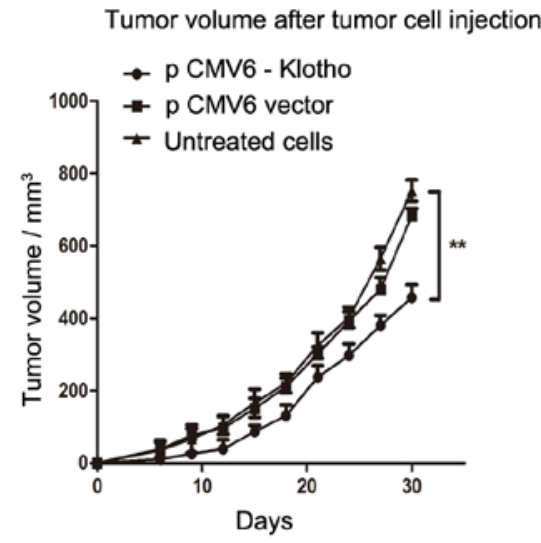

B

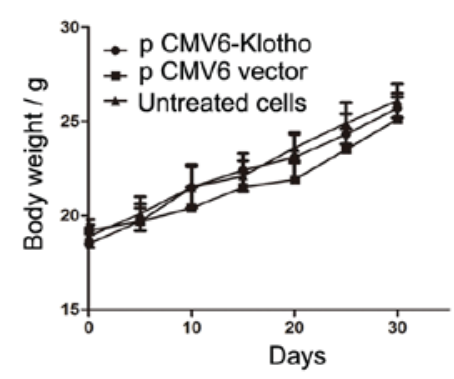

D

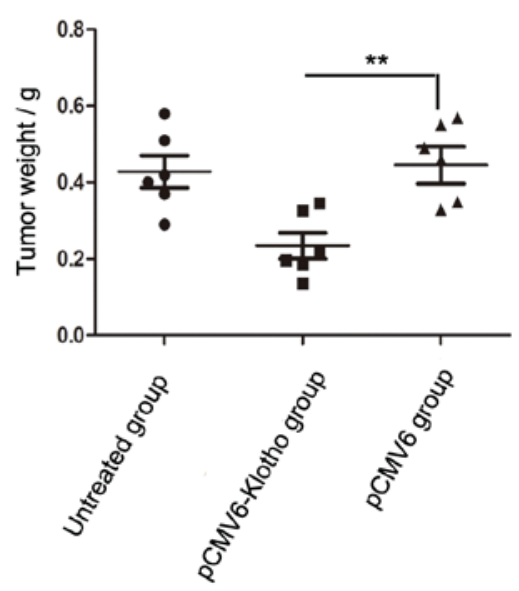

Figure 7. Overexpression of Klotho suppresses tumor growth in a murine model. (A) A2780 cells with low endogenous Klotho expression were transfected with pCMV6-Klotho vector or a control pCMV6 vector for $48 \mathrm{~h}$. Western blotting was used to determine the expression levels of Klotho in A2780 cells. $\beta$-actin was used as an internal inference. In a murine model, nude mice were injected subcutaneously with $3 \times 10^{5}$ tumor cells into the flank area ( $\mathrm{n}=6 / \mathrm{group}$ ). After tumor injection, body weight was weighed every 3 days. Mice were sacrificed after 1 month. (B) Body weight, (C) tumor volume and (D) tumor weight from the various groups are shown. ${ }^{* *} \mathrm{P}<0.01$, compared with the pCMV6 vector group.

vector-transfected cells $(\mathrm{P}<0.01$; Fig. 4B and $\mathrm{C})$. In addition, endogenous Klotho expression was suppressed by Klotho-specific shRNA. As expected, Klotho expression was suppressed in CaOV3 cells transfected with the shRNA, as detected by western blotting. Results of the MTT assay demonstrated that Klotho shRNA-transfected optical density $(490 \mathrm{~nm})$ values were increased compared with negative control shRNA-transfected cells or untreated cells $(\mathrm{P}<0.05$ and $\mathrm{P}<0.01$, compared with untreated cells; Fig. 4D-F). These data indicated that high levels of Klotho inhibited the proliferation of human ovarian cancer cells, and inhibiting the endogenous expression of Klotho may promote tumor cell growth in human ovarian cancer cells.

Plasma IL-6 and IL-1 $\beta$ levels are elevated in tumor-bearing mice. In order to detect the inflammatory responses in normal and tumor-bearing mice, inflammatory cytokine levels in the plasma were detected. The concentrations of plasma IL-6 and IL-1 $\beta$ were detected by ELISA. As shown in Fig. 5, mean plasma IL- 6 concentration was $63.0 \mathrm{pg} / \mathrm{ml}$ in tumor-bearing mice, which was significantly increased compared with in the wild type mice $(39.9 \mathrm{pg} / \mathrm{ml} ; \mathrm{P}<0.01)$. The levels of IL-1 $\beta$ showed a similar trend to IL- 6 . The concentration of IL-1 $\beta$ in the plasma of tumor-bearing mice was $46.43 \mathrm{pg} / \mathrm{ml}$, which was significantly increased compared with the mean value in wild type mice $(25.0 \mathrm{pg} / \mathrm{ml} ; \mathrm{P}<0.01)$. These results indicated that plasma IL- 6 and IL-1 $\beta$ levels are elevated in tumor-bearing mice, thus suggesting that the systemic inflammatory response was severe in tumor-bearing mice.

Aberrant Klotho expression contributes to systemic chronic inflammation in Klotho ${ }^{-/}$mice. In order to determine whether aberrant Klotho expression would induce or suppress systemic inflammation in mice, 8-week-old $\mathrm{Klotho}^{-/-}$mice and wild type mice with the same genetic background were used as an animal model. As presented in Fig. 6A, plasma IL-6 levels in $\mathrm{Klotho}^{-/ /}$mice were $65.0 \mathrm{pg} / \mathrm{ml}$, whereas plasma IL- 6 concentration was $32.0 \mathrm{pg} / \mathrm{ml}$ in the wild type mice with the same genetic background $(\mathrm{P}<0.05)$.

The mRNA expression levels of IL-6 were detected in various organs, including the liver, ovaries and kidneys (Fig. 6B-D). The results demonstrated that the expression levels of IL-6 were significantly elevated in the liver, ovaries and kidneys of the Klotho ${ }^{-/-}$mice compared with in the wild type mice $(\mathrm{P}<0.01)$. These data demonstrated that aberrant Klotho expression may contribute to systemic inflammation in $\mathrm{Klotho}^{-/}$mice.

Overexpression of Klotho suppresses tumor growth in a murine model. To assess the in vivo antitumor role of aberrant Klotho expression, A2780 human ovarian cancer cells were transfected with pCMV6-Klotho, and the stably transfected cells were screened. As shown in Fig. 7A, the expression of Klotho was markedly elevated in pCMV6-Klotho-transfected 
A2780 cells compared with in the PCMV6 vector-transfected cells or untreated cells. Mice were randomly divided into three groups ( $n=6 /$ group). The mice were subcutaneously challenged with $3 \times 10^{5}$ live stably transfected A2780 cells in the rear leg flank. A total of 10 days after the injection, tumors became gradually evident. The mice in each group were observed daily to monitor tumor volume. A total of 30 days after tumor injection, the mice were sacrificed and tumor weight was determined in each group. There was no significant difference in body weight between the three groups (Fig. 7B); however, tumor volume and tumor weight were significantly decreased in the PCMV6-Klotho group compared with in the pCMV6 vector group $(\mathrm{P}<0.01)$.

\section{Discussion}

Ovarian cancer is a common malignancy that affects the ovaries (23-25). Previous studies have demonstrated that Klotho is involved in the development and progression of several types of human tumor $(26,27)$; however, the effects of Klotho on ovarian cancer have not been clearly reported. The present study explored the role of Klotho in the progression of ovarian cancer, and investigated the molecular mechanism underlying the effects of Klotho during the progression of human ovarian tumors.

The present study detected Klotho expression in 120 ovarian cancer specimens and 78 normal ovarian specimens by western blotting and immunohistochemical analysis. The results demonstrated that Klotho acted as a tumor suppressor in human ovarian cancer. Notably, Klotho was highly expressed in normal control specimens; however, its expression was significantly reduced in $38.4 \%$ of specimens with ovarian cancers. Furthermore, reduced levels of Klotho were correlated with lower survival rates in patients with ovarian cancer $(\mathrm{P}=0.025)$. In addition, survival rate was not associated with age or metastasis. These results indicated that Klotho may serve as an indicator for the prognosis of patients with ovarian cancer.

The expression levels of Klotho were detected in seven human ovarian cancer cell lines by western blotting. Notably, $\mathrm{CaOV} 3$ cells were shown to have the highest levels of Klotho, CaOV4 and SKOV-3 cells had medium levels of Klotho protein, and four ovarian cancer cell lines, OVCA 432, OVCAR-5, OVCAR-8 and A2780, had almost no detectable levels of Klotho. Therefore, the ovarian cell lines with the highest and the lowest expression of Klotho served as a cell model. The results of an MTT assay demonstrated that overexpression of Klotho inhibited the proliferation of ovarian cancer cells, whereas suppression of Klotho promoted the growth of CaOV3 cells. These data suggested that Klotho may act as a tumor suppressor in human ovarian cancer cells.

An in vivo experiment in $\mathrm{Klotho}^{-/-}$mice demonstrated that IL-6 plasma concentration was significantly increased in $\mathrm{Klotho}^{-/}$mice compared with in wild type mice with the same genetic background. This result was consistent with the mRNA expression levels of IL-6 detected in the liver, ovaries and kidneys of Klotho ${ }^{-/}$mice. These data suggested that aberrant Klotho expression contributed to systemic inflammation. Notably, overexpression of Klotho suppressed tumor growth and tumor volume in a murine model, which was partly due to the inhibition of systemic inflammation and other tumor growth-related signaling pathways, such as Akt, ERK, insulin and Wnt signaling pathways.

In conclusion, the present study determined that aberrant Klotho expression contributed to systemic inflammation. Overexpression of Klotho suppressed tumor growth and tumor volume in a murine model, which was partly due to the inhibition of systemic inflammation.

\section{References}

1. Yahata T, Banzai C and Tanaka K; Niigata Gynecological Cancer Registry: Histology-specific long-term trends in the incidence of ovarian cancer and borderline tumor in Japanese females: A population-based study from 1983 to 2007 in Niigata. J Obstet Gynaecol Res 38: 645-650, 2012.

2. Maksimović M, Maksimović M, Gojnić M, Maksimović Z, Petković S, Ljubić A, Stefanović A and Jeremić K: Surgical treatment of ovarian cancer and early detection of venous thromboembolism. Eur J Gynaecol Oncol 32: 415-418, 2011.

3. Chen M, Jin Y, Bi Y, Li Y, Shan Y and Pan L: Prognostic significance of lymphovascular space invasion in epithelial ovarian cancer. J Cancer 6: 412-419, 2015.

4. Lloyd KL, Cree IA and Savage RS: Prediction of resistance to chemotherapy in ovarian cancer: A systematic review. BMC Cancer 15: 117, 2015.

5. Bacalbaşa $\mathrm{N}$ and Popescu I: Ovarian cancer liver metastases - should we apply the principle of optimal cytoreduction to the liver? A review. Hepatogastroenterology 62: 355-357, 2015.

6. Rooth C: Ovarian cancer: Risk factors, treatment and management. Br J Nurs 22: S23-S30, 2013.

7. Liu XH, Man YN and Wu XZ: Recurrence season impacts the survival of epithelial ovarian cancer patients. Asian Pac J Cancer Prev 15: 1627-1632, 2014.

8. Dërmaku-Sopjani M, Kolgeci S, Abazi S and Sopjani M: Significance of the anti-aging protein Klotho. Mol Membr Biol 30: 369-385, 2013.

9. Zeng Y, Wang PH, Zhang M and Du JR: Aging-related renal injury and inflammation are associated with downregulation of Klotho and induction of RIG-I/NF- $\mathrm{KB}$ signaling pathway in senescence-accelerated mice. Aging Clin Exp Res 28: 69-76, 2016.

10. Banerjee S, Zhao Y, Sarkar PS, Rosenblatt KP, Tilton RG and Choudhary S: Klotho ameliorates chemically induced endoplasmic reticulum (ER) stress signaling. Cell Physiol Biochem 31: 659-672, 2013.

11. Huang CL: Regulation of ion channels by secreted Klotho. Adv Exp Med Biol 728: 100-106, 2012.

12. Kuro-o M: Klotho and aging. Biochim Biophys Acta 1790: 1049-1058, 2009.

13. Lojkin I, Rubinek T, Orsulic S, Schwarzmann O, Karlan BY, Bose S and Wolf I: Reduced expression and growth inhibitory activity of the aging suppressor klotho in epithelial ovarian cancer. Cancer Lett 362: 149-157, 2015.

14. Wolf I, Levanon-Cohen S, Bose S, Ligumsky H, Sredni B, Kanety H, Kuro-o M, Karlan B, Kaufman B, Koeffler HP and Rubinek T: Klotho: A tumor suppressor and a modulator of the IGF-1 and FGF pathways in human breast cancer. Oncogene 27: 7094-7105, 2008.

15. Zhou X and Wang X: Klotho: A novel biomarker for cancer. J Cancer Res Clin Oncol 141: 961-969, 2015.

16. Xie B, Chen J, Liu B and Zhan J: Klotho acts as a tumor suppressor in cancers. Pathol Oncol Res 19: 611-617, 2013.

17. Lee J, Jeong DJ, Kim J, Lee S, Park JH, Chang B, Jung SI, Yi L, Han Y, Yang Y, et al: The anti-aging gene KLOTHO is a novel target for epigenetic silencing in human cervical carcinoma. Mol Cancer 9: 109, 2010.

18. Lu L, Katsaros D, Wiley A, de la Longrais IA, Puopolo M and Yu H: Klotho expression in epithelial ovarian cancer and its association with insulin-like growth factors and disease progression. Cancer Invest 26: 185-192, 2008.

19. Saleem M, Maddodi N, Abu Zaid M, Khan N, bin Hafeez B, Asim M, Suh Y, Yun JM, Setaluri V and Mukhtar H: Lupeol inhibits growth of highly aggressive human metastatic melanoma cells in vitro and in vivo by inducing apoptosis. Clin Cancer Res 14: 2119-2127, 2008. 
20. Adhami VM, Siddiqui IA, Ahmad N, Gupta S and Mukhtar H: Oral consumption of green tea polyphenols inhibits insulin-like growth factor-I-induced signaling in an autochthonous mouse model of prostate cancer. Cancer Res 64: 8715-8722, 2004.

21. Spinner DM: MTT growth assays in ovarian cancer. Methods Mol Med 39: 175-177, 2001.

22. Sargent J, Elgie A, Taylor CG, Wilson J, Alton P and Hill JG: The identification of drug resistance in ovarian cancer and breast cancer: Application of the MTT assay. Contrib Gynecol Obstet 19: 64-75, 1994.

23. Ebell MH, Culp M, Lastinger K and Dasigi T: A systematic review of the bimanual examination as a test for ovarian cancer. Am J Prev Med 48: 350-356, 2015.
24. Ozga M, Aghajanian C, Myers-Virtue S, McDonnell G, Jhanwar S, Hichenberg S and Sulimanoff I: A systematic review of ovarian cancer and fear of recurrence. Palliat Support Care 13: 1771-1780, 2015.

25. Iżycka N, Lubin J, Markowska A and Markowska J: Late recurrence of ovarian cancer: A literature review and description of two cases. Eur J Gynaecol Oncol 36: 351-353, 2015.

26. Martín-Núñez E, Donate-Correa J, Muros-de-Fuentes M, Mora-Fernández C and Navarro-González JF: Implications of Klotho in vascular health and disease. World J Cardiol 6: 1262-1269, 2014.

27. Wang Y, Chen L, Huang G, He D, He J, Xu W, Zou C, Zong F, Li Y, Chen B, et al: Klotho sensitizes human lung cancer cell line to cisplatin via PI3k/Akt pathway. PloS One 8: e57391, 2013. 